\title{
Rheological Properties of Rodlike Polymers in Solution III. Transient and Steady-State Studies on Nematic Solutions
}

\author{
Y. EINAGA, ${ }^{\dagger}$ G. C. BERRY, ${ }^{*}$ and S. G. $\mathrm{CHU}^{\dagger \dagger}$ \\ Department of Chemistry, Carnegie-Mellon University, \\ Pittsburgh, PA 15213, U.S.A.
}

(Received August 20, 1984)

\begin{abstract}
Rheological studies are reported on nematic solutions of the rodlike macroion poly(14-phenylene-2,6-benzobisthiazole), PBT. The data reported include the steady-state viscosity $\eta_{\kappa}$ and recoverable compliance $R_{\kappa}$ as functions of the shear rate $\kappa$, the creep compliance $J_{\sigma}(t)$ and the recoverable compliance $R_{\sigma}(t)$ determined with shear stress $\sigma$, and the viscosity growth function $\eta_{\kappa}(t)$ determined with shear rate $\kappa$. The nematic solutions did not exhibit true linear viscoelastic behavior in the range studied $\left(\sigma \geq 14 \mathrm{~Pa}\right.$ and $\left.\kappa \geq 10^{-4} \mathrm{~s}^{-1}\right)$. Neither was any "yield behavior" observed over this range. The steady-state parameters are discussed in terms of a single-integral constitutive equation of the BKZ-type in which the relaxation times are estimated from data on $R_{\sigma}(t)$ at small $\sigma$.
\end{abstract}

KEY WORDS Flow Birefringence / Nematic Solution / Rodlike Polymer / Rheology / Nonlinear Creep /

In Parts 1 and 2 of this series, ${ }^{1,2}$ the rheological and rheo-optical properties of mesogenic solutions of rodlike macrions are reported for conditions of concentration, rod length and temperature for which the solutions are optically isotropic. Steadystate and transient properties are reported in parts I and II, respectively, and discussed in terms of a single-integral constitutive equation and molecular theories. Here, some steady-state and transient rheological properties will be presented for nematic solutions of two samples of one of the polymers studied in Parts I and II, namely, poly $(1,4-$ phenylene-2,6-benzobisthiazole), PBT. The data to be reported include the steady-state viscosity $\eta_{\kappa}$ and recoverable compliance $R_{\kappa}$ as functions of the shear rate $\kappa$, the creep compliance $J_{\sigma}(t)$ and recoverable compliance $R_{\sigma}(t)$ determined with shear stress $\sigma$, and the viscosity growth function $\eta_{\kappa}(t)$ determined with shear rate $\kappa$.
The transient function (i.e., $J_{\sigma}(t), R_{\sigma}(t)$ and $\eta_{\kappa}(t)$ ) are defined in Part II. In Part II, the limiting values $R_{0}(t)$ of $R_{\kappa}(t)$ at small $\sigma$, and $\eta_{0}(t)$ of $\eta_{\kappa}(t)$ at small $\kappa$ were expressed in terms of retardation and relaxation times, respectively, as ${ }^{3}$ :

$$
\begin{aligned}
& R_{0}(t)=R_{0}-\sum_{1}^{n-1} R_{i} \exp \left(-t / \lambda_{i}\right) \\
& \eta_{0}(t)=\eta_{0}-\sum_{1}^{n} \eta_{i} \exp \left(-t / \tau_{i}\right)
\end{aligned}
$$

where, of course, the set of $\eta_{i}$ and $\tau_{i}$ may be determined from the set of $R_{i}$ and $\lambda_{i}$ together with $R_{0}$ and $\tau_{\mathrm{c}}=R_{0} \eta_{0}$, and vice versa. In Part II it was found that a single-integral relation for the shear stress $\sigma(t)$ given by ${ }^{2,4}$

$$
\sigma(t)=\sum \eta_{i} \tau_{i}^{-2} \int_{0}^{\infty} \Delta \gamma(t, u) F[\Delta \gamma(t, u)] \exp \left(-u / \tau_{i}\right) \mathrm{d} u
$$

\footnotetext{
* To whom correspondence should be sent.

† Permanent address: Department of Macromolecular Science, Osaka University, Osaka, Japan.

${ }_{\dagger}$ Permanent address: Hercules Research Laboratory, Wilmington, DE, U.S.A.
} 
could be used to predict $\eta_{\kappa}$ and $R_{\kappa}$. Here $\Delta \gamma(t, u)=$ $\gamma(t)-\gamma(t-u)$ and $F(\gamma)$ is given by the expression

$$
F(\gamma)=\exp \left[-m\left(|\gamma|-\gamma^{\prime}\right) / \gamma^{\prime \prime}\right]
$$

where $m$ is zero for $|\gamma|<\gamma^{\prime}$ and unity otherwise, with $\gamma^{\prime}$ and $\gamma^{\prime \prime}$ material parameters. Of course, if $F(\gamma)$ is unity (i.e., $|\gamma|<\gamma^{\prime}$, then eq 3 reduces to the result for the linear Boltzmann constitutive equation. With eq 3 and $4,{ }^{4}$

$$
\begin{gathered}
\eta_{\kappa}=\sum \eta_{i}\left(1-q_{\kappa, i}\right) \\
\eta_{0} \eta_{\kappa} R_{\kappa} \approx \sum \eta_{i} \tau_{i}\left[1-q_{\kappa, i} \exp \left(-\eta_{\kappa} R_{\kappa} / \tau_{i}\right)\right]
\end{gathered}
$$

where

$$
q_{\kappa, i}=\left(1+\alpha f_{i}^{2}-f_{i}\right) \exp \left(-g_{i}\right)
$$

with $f_{i}^{-1}=1+\alpha g_{i}^{-1}, g_{i}=\gamma^{\prime} / \tau_{i} \kappa$ and $\alpha=\gamma^{\prime} / \gamma^{\prime \prime}$. These relations provided satisfactory fits to data on $\eta_{\kappa}$ and $R_{\kappa^{\prime}}$ given experimental data on $\eta_{i}$ and $\tau_{i}$ for the isotropic solutions. Based on studies ${ }^{2}$ of $J_{\sigma}(t), \gamma^{\prime}$ was found to be inversely proportional to $c$. Mechanistic models with results similar to eq 3 and 4 are discussed in Part II. ${ }^{2,5,6}$ These differ from experiment principally in predicting a far narrower distribution of $\tau_{i}$ (or $\lambda_{i}$ ) than found in our studies.

For the data in Pars I and II, dependence of $\eta_{0}$ on $c$ and the rodlength $L$ was given by the expression $^{1,2,5}$

$$
\eta_{0} / \eta_{\mathrm{s}}=K N_{\mathrm{A}}^{2} \dot{M}[\eta] \alpha^{* 3} X^{3}(1-B X)^{-2}
$$

for $X<1$, where $X=c L / M_{\mathrm{L}} \alpha^{*}$, with $M_{\mathrm{L}}=M / L,[\eta]$ is the intrinsic viscosity and $\alpha^{*} / B$ and $K$ are parameters of the models. Of the latter, $B$ is expected to be nearly unity, ${ }^{5} \alpha^{*} M_{\mathrm{L}} / L$ is expected to be nearly equal to the concentration $c_{\mathrm{c}}$ 'required to form a

\begin{tabular}{|c|c|c|c|c|}
\hline \multirow[t]{2}{*}{ Polymer } & {$[\eta]$} & $L_{\eta}^{\mathrm{a}}$ & $\begin{array}{c}\text { Range } \\
\qquad w\end{array}$ & $\begin{array}{l}\text { Range } \\
\quad T\end{array}$ \\
\hline & $\mathrm{mlg}^{-1}$ & $\mathrm{~nm}$ & $\mathrm{~g} \mathrm{~kg}^{-1}$ & K \\
\hline PBT-62 & 2,650 & 170 & $15.0-82$ & $272-353$ \\
\hline PBT-53 & 1,400 & 120 & $25.5-42.7$ & $283-333$ \\
\hline
\end{tabular}
nematic phase, and the original calculation ${ }^{5}$ gave $K \sim 1$. With the data reported in Parts I and II, the former two predictions are verified, but

Table I. Polymers used in this study

a $L_{\eta}=M_{\eta} / M_{\eta}$, where $M_{\mathrm{L}}=220$ dalton $\mathrm{nm}^{-1}$ and $M_{\eta}=$ $\left([\eta] / K_{\eta}\right)^{1.8}$ with $M_{\mathrm{L}}^{1.8} K_{\eta}=0.26 \mathrm{mlg}^{-1}$.
$K \approx 1.5 \times 10^{-4}$, in better agreement with revised estimates. $^{7}$

\section{EXPERIMENTAL}

The materials and methods used are described fully in Parts I and II. The polymers used in this study are listed in Table I. Sample PBT-53 was studied in solution in methane sulfonic acid (MSA), and PBT-62 was studied in solution in MSA containing 3 weight percent chlorosulfonic acid. The range of the weight fraction $w$ and temperature $T$ spanned in the studies is given in Table $\mathrm{I}$, and shown schematically in Figures 1 and 2. With PBT-62, only steady-state data were obtained, e.g., $\eta_{\kappa}$ and $R_{\kappa}$. With PBT-53, in addition to the steady-state data, experiments included $J_{\sigma}(t), R_{\sigma}(t)$ and $\eta_{\kappa}(t)$.

\section{RESULTS}

The systematic effects observed on $\eta_{\kappa}$ and $R_{\kappa}$ are illustrated in Figures 3 and 4, respectively, for several concentrations of PBT- 62 for $T \approx 60^{\circ} \mathrm{C}$ and for a solution of PBT-53 for $T \approx 23^{\circ} \mathrm{C}$. The limiting values $\eta_{0}$ and $R_{0}$ obtained for $\eta_{\kappa}$ and $R_{\kappa^{\prime}}$ respectively, at small $\kappa$ are listed in Table II.

As may be seen in Figures 3 and 4, in some cases $\eta_{\kappa}$ did not go to a limiting value at small $\kappa$ with the nematic solutions- - this feature is discussed further in the next section. In such cases, $R_{\kappa}$ did tend to a constant value $R_{0}$ at small $\kappa$, and usually a range of $\kappa$ existed for which $\eta_{\kappa}$ was essentially a constant, designated $\eta_{\mathrm{p}}$. Values of $R_{0}$ and $\eta_{\mathrm{P}}$ are entered in Table II for the nematic fluids.

The data on $\eta_{0}$ are reduced to $24^{\circ} \mathrm{C}$ using $\partial \ln \eta / \partial T^{-1}$ given in Part II. For the isotropic solution, $\partial \ln \eta / \partial T^{-1}$ is about equal to $\partial \ln \eta_{\mathrm{s}} w_{\mathrm{c}}^{3} / \partial T^{-1}$, as expected with eq 8 , where $w_{\mathrm{c}}$ is the concentration required to form a stable nematic phase. For the nematic solution, $\partial \ln \eta / \partial T^{-1}$ is smaller, and more nearly equal to $\partial \ln \eta_{\mathrm{s}} / \partial T^{-1}$. Consequently, data on $\eta_{\mathrm{P}}$ are reduced to $24^{\circ} \mathrm{C}$ using $\partial \ln \eta_{\mathrm{s}} / \partial T^{-1}$.

Additional data on $\eta_{\kappa}$ and $R_{\kappa}$ for PBT-62 and data for PBT-53 are given in Figures 5-11, in plots of the reduced functions $\eta_{\mathrm{k}} / \eta_{0}\left(\eta_{\kappa} / \eta_{\mathrm{P}}\right)$ and $R_{\kappa} / R_{0}$ versus $\eta_{0} R_{0} \kappa$ (or $\eta_{\mathrm{P}} R_{0} \kappa$ ). Except for data on nematic solutions with $\eta_{\mathrm{P}} R_{0} \kappa<1$, these plots are independent of $T$ for a given concentration.

Data on $J_{\sigma}(t)$ versus $t$ are given in Figure 12 for a nematic solution of PBT-53 $\left(w=0.0323\right.$ at $\left.19^{\circ} \mathrm{C}\right)$. 
The behavior is similar to that reported for an isotropic solution of PBT-53 $\left(w=0.0255\right.$ at $\left.30^{\circ} \mathrm{C}\right)$ discussed in Part II. As with the latter, $J_{\sigma}(t)$ is found to equal the limiting behavior $J_{0}(t)$ for small $\sigma$ provided the strain $\gamma(t)=\sigma J_{\sigma}(t)$ is less than a critical value $\gamma^{*}$, as indicated on Figure 12. The recoverable compliance function $R_{\sigma}(t)$ obtained following steady-state deformation is also shown in Figure 12 for one shear stress $(\sigma=14.6$ lowing steady-state

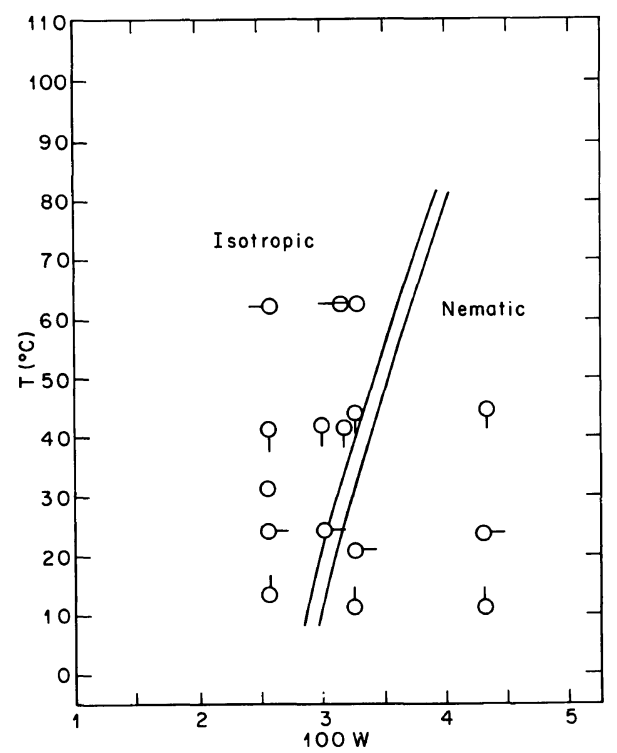

Figure 1. Schematic diagram showing the concentration (weight fraction $w$ ) and temperature used in rheological studies on solutions of PBT-53. Regions of isotropic and nematic solutions are indicated in the diagram. deformation is also shown in Figure 12 for one shear stress $(\sigma=14.6 \mathrm{~Pa})$.

Data on $\sigma(t)$ as a function of the strain $\kappa t$ in a stress growth experiment are given in Figures 13 and 14. In these experiments $\kappa$ is constant for $\kappa t$

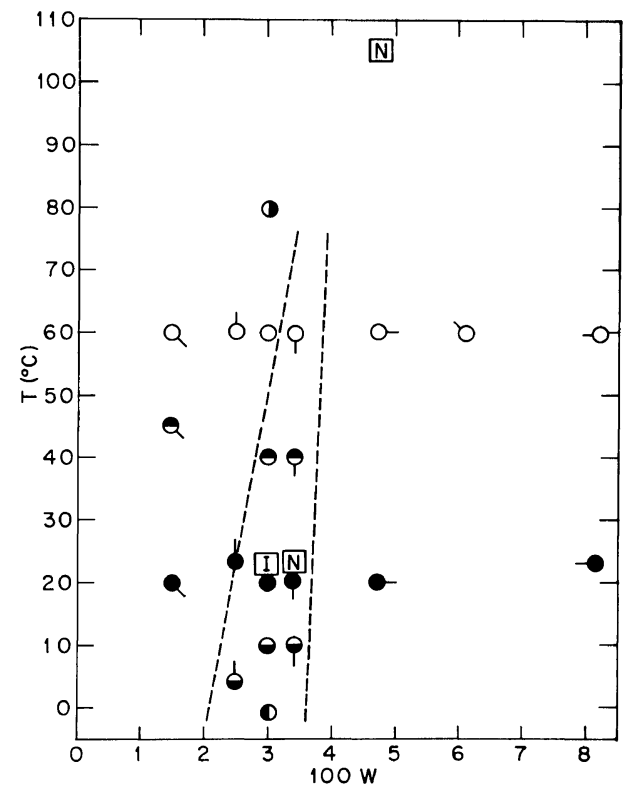

Figure 2. Schematic diagram showing the concentrations (weight function $w$ ) and temperatures used in rheological studies on solutions of PBT-62. Conditions of $w$ and $T$ for which solutions were observed to be isotropic or nematic are indicated by $\Pi$ or $\mathbb{N}$, respectively. Data for conditions to the left and right sides of the dashed line are given in Figures 5 and 9, respectively.

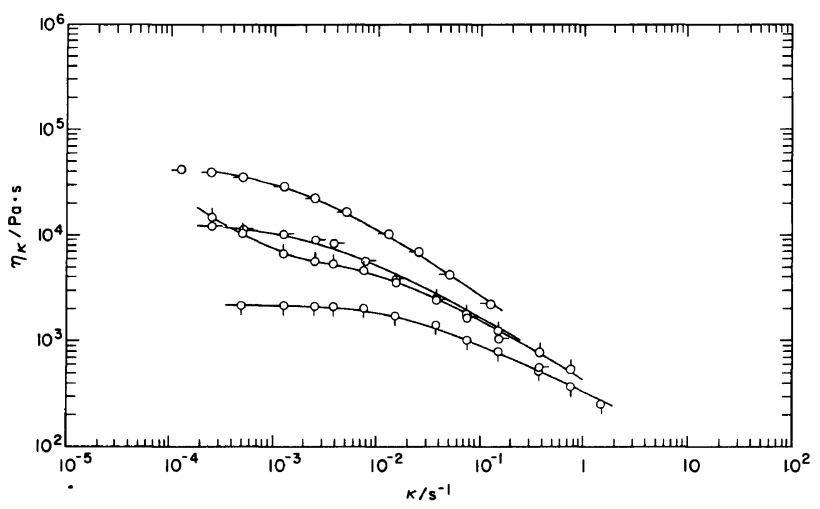

Figure 3. The steady-state viscosity of $\eta_{\kappa}$ for solutions of PBT-53 with $T \simeq 20^{\circ} \mathrm{C}$. The symbols indicate $w / \mathrm{g} \mathrm{kg}^{-1}$ of $25.5, \bigcirc ; 29.4,-\bigcirc ; 32.3, \bigcirc$ and $42.7, \bigcirc-$. 


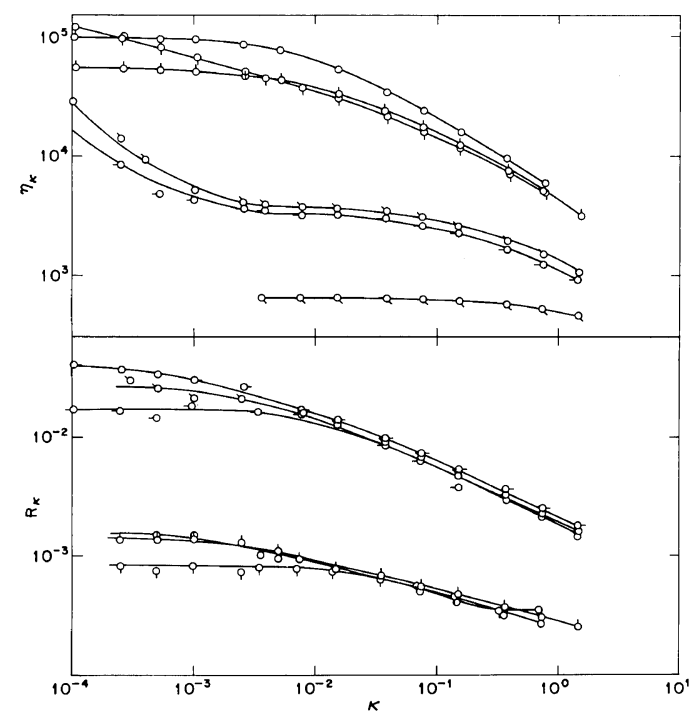

Figure 4. The steady-state viscosity $\eta_{\kappa}$ and recoverable compliance $R_{\kappa}$ for solutions of PBT-62 with $T \approx 60^{\circ} \mathrm{C}$. The symbols indicate $w$ as in Figure 2.

greater than about 0.3 , but owing to instrumental limitations, $\gamma(t) \leq \kappa t$ for smaller $\kappa t$. The data reveal a maximum in $\sigma(t)$ with increasing $t$ such that $\partial \sigma(t) /$ $\partial t$ is zero for $\kappa t$ equal to a value $\gamma^{+}$that is nearly independent of $\kappa$, and smaller than $\gamma^{*}$ determined from $J_{\sigma}(t)$. Values of $\gamma^{*}$ and $\gamma^{+}$determined for isotropic and nematic solutions over a range of $c$ are given in Figure 15.

\section{DISCUSSION}

The decrease of "the viscosity" on the formation of the nematic phase is one of the most characteristic features noted for mesogenic polymer solutions. ${ }^{8}$ The value of $\eta_{\kappa}$ usually referred to in this correlation is $\eta_{\mathrm{P}}$ defined above. Values of $\eta_{\mathrm{P}}$ determined here are given in Figure 16, along with values of $\eta_{0}$ for isotropic solutions reported in Parts I and II; as mentioned above, the latter are well represented by eq 8 . In a theoretical treatment related to that used to derive eq 8 , Doi has calculated the steady-state viscosity $\eta_{\text {aniso }}$ for a "uniform" fluid, in a "weak velocity gradient." 9 The fluid is characterized by an order parameter $S$ related to the ratio $c / c_{\mathrm{c}}$ :

$$
S=\frac{1}{4}+\frac{3}{4}\left(1-\frac{8}{9} \frac{c_{\mathrm{c}}}{c}\right)^{1 / 2}
$$

Table II. Rheological parameters for solutions of rodlike polymers

\begin{tabular}{|c|c|c|c|c|}
\hline \multirow{2}{*}{ Polymer } & $w$ & $T$ & $\eta_{0} A^{-1}$ & \multirow{2}{*}{$R_{0} c R T / M_{\eta}^{\mathrm{b}}$} \\
\hline & $\mathrm{g} \mathrm{kg}^{-1}$ & $\mathrm{~K}$ & $\mathrm{kPas}^{\mathrm{a}}$ & \\
\hline \multirow[t]{9}{*}{53} & $25.5(\mathrm{I})^{\mathrm{c}}$ & 333 & 1.89 & $(60)$ \\
\hline & $25.5(\mathrm{I})$ & 313 & 1.83 & $(60)$ \\
\hline & $25.5(\mathrm{I})$ & 303 & 1.73 & (62) \\
\hline & $25.5(\mathrm{I})$ & 296 & 2.10 & 64 \\
\hline & $25.5(\mathrm{I})$ & 286 & 1.89 & 69 \\
\hline & $29.4(\mathrm{I})$ & 313 & 7.15 & 70 \\
\hline & 29.4 (I) & 296 & 7.64 & 78 \\
\hline & 29.4 (I) & 285 & 6.80 & 107 \\
\hline & $32.3(\mathrm{I})$ & 333 & 4.83 & 102 \\
\hline & $32.3(\mathrm{~N})$ & 316 & 4.05 & 97 \\
\hline & $32.3(\mathrm{~N})$ & 294 & 4.34 & 112 \\
\hline & $32.3(\mathrm{~N})$ & 283 & 6.90 & 130 \\
\hline & $42.7(\mathrm{~N})$ & 316 & 12.3 & 190 \\
\hline & $42.7(\mathrm{~N})$ & 296 & 11.0 & 180 \\
\hline & $42.7(\mathrm{~N})$ & 284 & 9.6 & 172 \\
\hline \multirow[t]{21}{*}{62} & $15.0 \cdot(\mathrm{I})$ & 333 & 0.28 & 18 \\
\hline & $15.0(\mathrm{I})$ & 318 & 0.31 & 17 \\
\hline & $15.0(\mathrm{I})$ & 293 & -0.27 & 21 \\
\hline & $25.2(\mathrm{I})$ & 333 & 22.8 & 42 \\
\hline & $25.2(\mathrm{I})$ & 297 & 41.0 & 31 \\
\hline & $25.2(\mathrm{I})$ & 277 & 52.0 & 18 \\
\hline & $30.0(\mathrm{I})$ & 353 & 45.6 & 63 \\
\hline & $30.0(\mathrm{I})$ & 333 & 41.7 & 53 \\
\hline & $30.0(\mathrm{I})$ & 313 & 60.4 & 44 \\
\hline & $30.0(\mathrm{I})$ & 293 & 74.7 & 35 \\
\hline & $30.0(\mathrm{I})$ & 283 & 86.4 & 30 \\
\hline & 30.0 (I) & 272 & 71.1 & 23 \\
\hline & $34.3(\mathrm{I})$ & 333 & 13.2 & 31 \\
\hline & $34.3(\mathrm{I})$ & 313 & 13.1 & 21 \\
\hline & $34.3(\mathrm{~N})$ & 293 & 3.1 & 33 \\
\hline & $34.3(\mathrm{~N})$ & 283 & 3.0 & 25 \\
\hline & $47.3(\mathrm{~N})$ & 333 & 0.61 & 940 \\
\hline & $47.3(\mathrm{~N})$ & 313 & 0.53 & 970 \\
\hline & $61.1(\mathrm{~N})$ & 333 & 0.94 & 2030 \\
\hline & $82.0(\mathrm{~N})$ & 333 & 0.83 & 3630 \\
\hline & $82.0(\mathrm{~N})$ & 297 & 0.47 & 3640 \\
\hline
\end{tabular}

a $A=\exp E\left(T^{-1}-T_{\mathrm{r}}^{-1}\right)$ with $T_{\mathrm{r}}=297 \mathrm{~K}$ and $E / \mathrm{K}$ equal to 4065 for isotropic solutions, and 2800 for nematic solutions, see the text.

b $R_{0}$ in parenthesis calculated as $\tau_{\mathrm{c}} / \eta_{0}$ with $\tau$ determined from fit of $\eta_{\kappa} / \eta_{0}$ versus $\tau_{\mathrm{c}} \kappa$.

c Symbols I and $\mathrm{N}$ indicate solutions which were isotropic or nematic at rest, respectively.

for $c / c_{c}>8 / 9$ and zero for smaller $c$. With this model, for $c>c_{\mathrm{c}}$,

$$
\eta_{\text {aniso }}=\eta_{\text {iso }}\left(c_{\mathrm{c}}\right) D(S)
$$


Rheological Properties of Rodlike Polymers in Solution III.

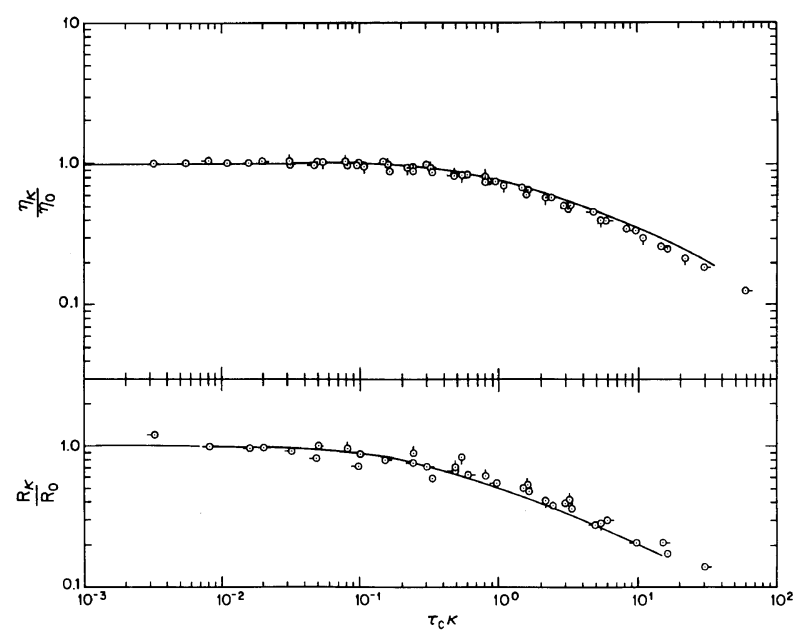

Figure 5. $\eta_{\kappa} / \eta_{0}$ and $R_{\kappa} / R_{0}$ versus $R_{0} \eta_{0} \kappa$ for isotropic solutions of PBT-53 ( $w=25.5 \mathrm{~g} / \mathrm{kg}$, with symbols as in Figure 1). The curves for $\eta_{\kappa} / \eta_{0}$ and $R_{\kappa} / R_{0}$ represent eq 5 and 6, respectively, with the $\tau_{i}$ and $\eta_{i}$ in Table III.

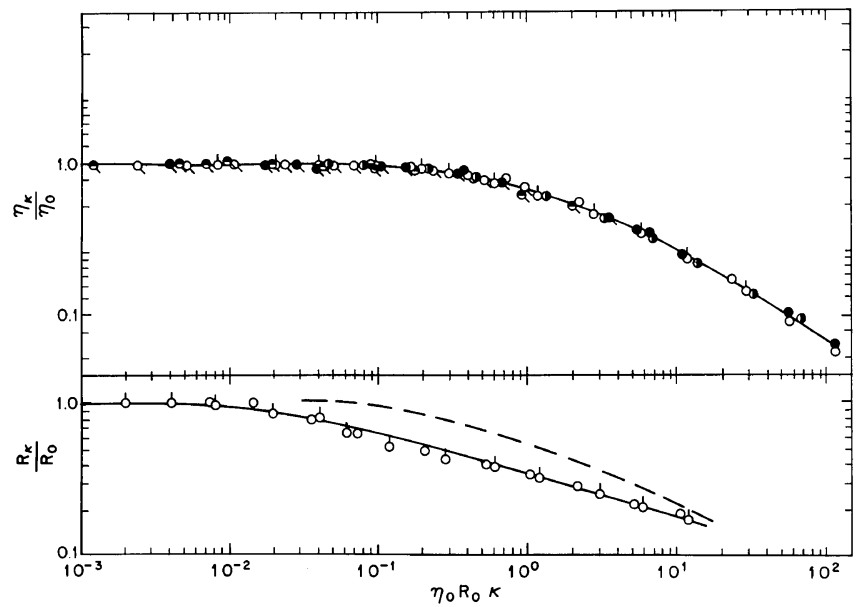

Figure 6. $\eta_{\kappa} / \eta_{0}$ and $R_{\kappa} / R_{0}$ versus $R_{0} \eta_{0} \kappa$ for isotropic solutions of PBT-62 (symbols as in Figure 2). The dashed curve represents $R_{\kappa} / R_{0} v s . \tau_{\mathrm{c}} \kappa$ from Figure 5.

where $\eta_{\text {iso }}\left(c_{\mathrm{c}}\right)$ is the viscosity of an isotropic solution at concentration $c_{\mathrm{c}}$, given by eq 8 and $D(S)$ decreases with increasing $S$ :

$D(S)=(1-S)\left(\frac{1+S}{1+2 S}\right)^{2} \frac{1+3 S / 2}{(1+S / 2)^{2}} \approx(1-S) /(1+S / 2)^{2}$

With eq $10, \eta_{\text {aniso }} / \eta_{\text {iso }}\left(c_{\mathrm{c}}\right)$ is expected to depend on $c / c_{\mathrm{c}}$ through the dependence of $S$ on $c / c_{\mathrm{c}}$. The data in Figure 16 conform qualitatively to eq 10 in that
$\eta_{\mathrm{P}}$ decreases from $\eta_{\text {iso }}\left(c_{\mathrm{c}}\right)$ with increasing $c / c_{\mathrm{c}}$, but $\eta_{\mathrm{P}}$ appears to level and then increase with larger $c / c_{\mathrm{c}}$, whereas $D(S)$ decreases monotonically with increasing $c / c_{\mathrm{c}}$.

The continuous increase of $\eta_{\kappa}$ with decreasing $\kappa$ in experiments observed with most of the nematic solutions is similar to behavior reported previously in our laboratory ${ }^{10}$ for nematic solutions of poly(1,4-phenylene-2,6-benzobisoxazole) and poly(1,4-phenylene terephthalate), as well as by a num- 


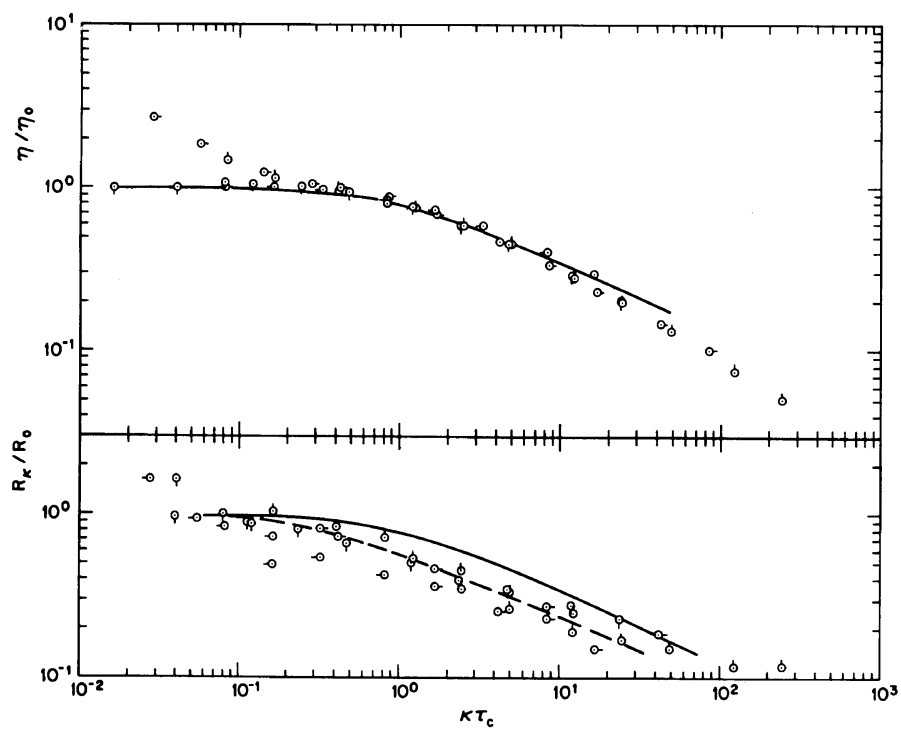

Figure 7. $\eta_{\kappa} / \eta_{0}$ (or $\eta_{\kappa} / \eta_{0}$ ) and $R_{\kappa} / R_{0}$ versus $R_{0} \eta_{0} \kappa$ (or $R_{0} \eta_{\mathrm{P}} \kappa$ ) for isotropic (or nematic) solutions of PBT$53\left(w=32.3 \mathrm{~g} \mathrm{~kg}^{-1}\right.$, with symbols as in Figure 1). The solid curves for $\eta_{\kappa} / \eta_{0}$ and $R_{\kappa} / R_{0}$ represent eq 5 and 6 , respectively with the $\tau_{i}$ and $\eta_{i}$ given in Table III. The dashed curve shows $R_{0}(t) / R_{0}$ versus $\tau_{\mathrm{c}} / t$.

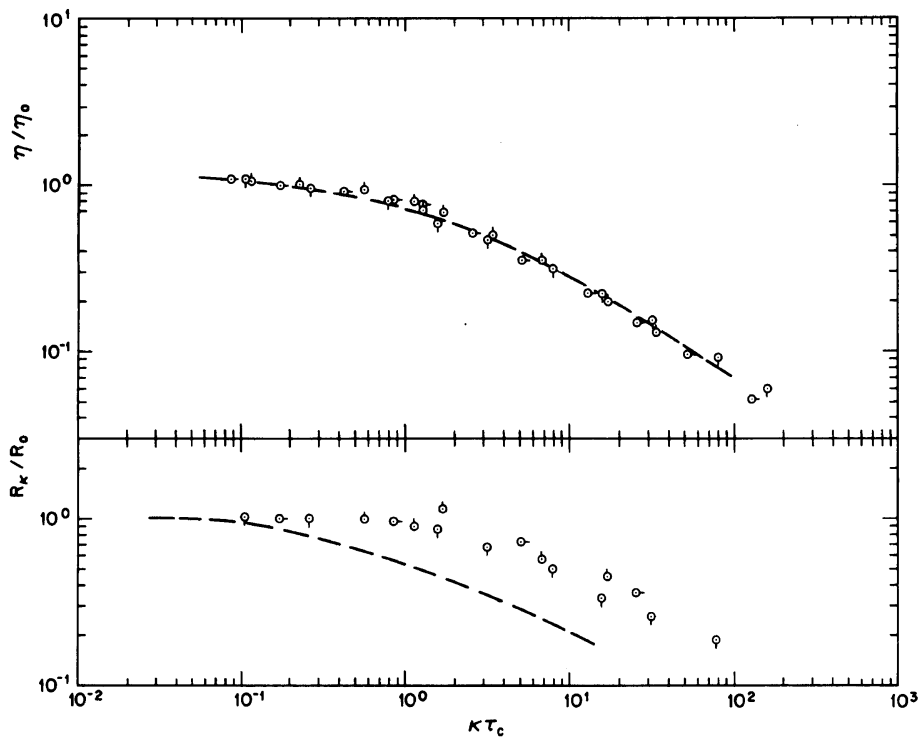

Figure 8. $\eta_{\kappa} / \eta_{\mathrm{P}}$ and $R_{\kappa} / R_{0}$ versus $R_{0} \eta_{0} \kappa$ for nematic solutions of PBT-53 ( $w=42.7 \mathrm{~g} \mathrm{~kg}^{-1}$, with symbols as in Figure 1). The dashed curves represent the curves given in Figure 7.

ber of others (see the discussion in ref 8 ). The behavior is not always observed over the range of $\kappa$ studied, ${ }^{8}$ and indeed, is not observed here with the nematic solution of PBT-53 with $w=42.7 \mathrm{~g} \mathrm{~kg}^{-1}$. The transient behavior discussed in the following for $J_{\sigma}(t)$ at small $\sigma$ and $\eta_{\kappa}(t)$ at small $\kappa$ displays unusual behavior in the range for which $\eta_{\kappa}$ increases with increasing $\kappa$.

As shown in Figures 13 and 14, in a stress-growth experiment for the nematic solutions studied, for 
Rheological Properties of Rodlike Polymers in Solution III.

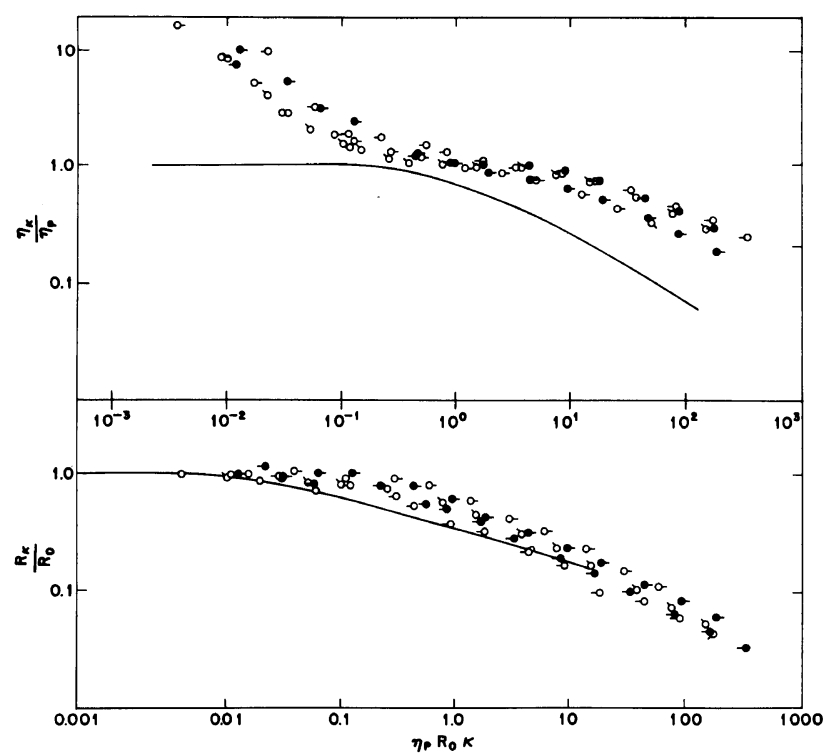

Figure 9. $\eta_{\kappa} / \eta_{\mathrm{P}}$ and $R_{\kappa} / R_{0}$ versus $R_{0} \eta_{\mathrm{P}} \kappa$ for nematic solutions of PBT-62 (symbols as in Figure 2). The curves represent the behavior shown in Figure 6.

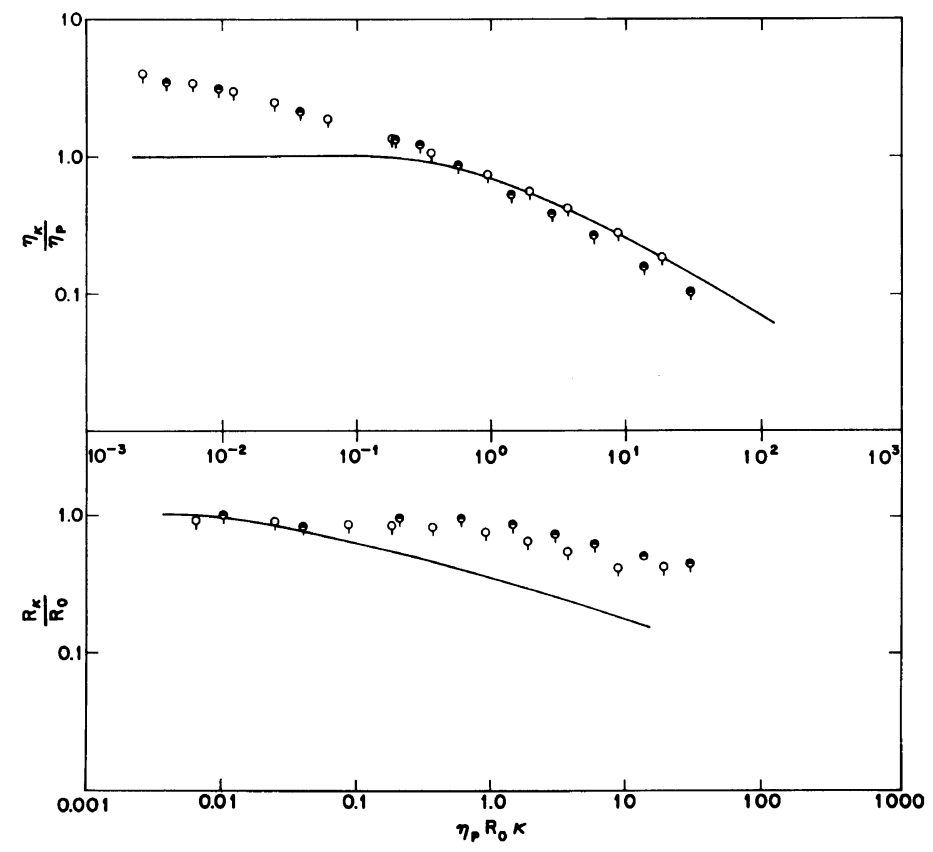

Figure 10. $\eta_{\kappa} / \eta_{\mathrm{P}}$ and $R_{\kappa} / R_{0}$ versus $R_{0} \eta_{\mathrm{P}} \kappa$ for solutions of PBT-62 (symbols as in Figure 2). The curves represent the behavior shown in Figure 6.

small $\kappa$ the stress $\sigma(t)$ increases monotonically with increasing strain $\kappa t$. In the creep behavior obtained with a nematic solution (Figure 12), the strain $\gamma(t)=$ $\sigma J_{\sigma}(t)$ increases monotonically with increasing $t$, so that at large $t, \gamma(t) \approx \sigma t / \eta_{\kappa}$ and $\partial \gamma(t) / \partial t$ is a constant. In neither case is there any indication of solid-like 


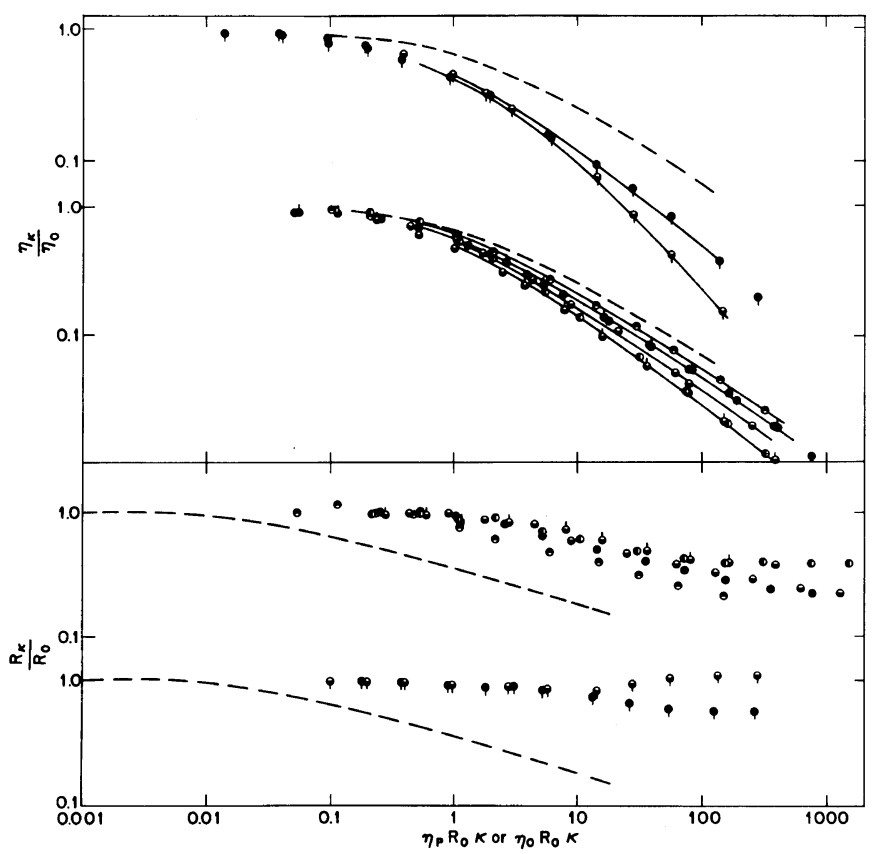

Figure 11. $\eta_{\kappa} / \eta_{0}$ and $R_{\kappa} / R_{0}$ versus $R_{0} \eta_{0} \kappa$ for solutions of PBT-62 (symbols as in Figure 2). The dashed curves represent the behavior shown in Figure 6.

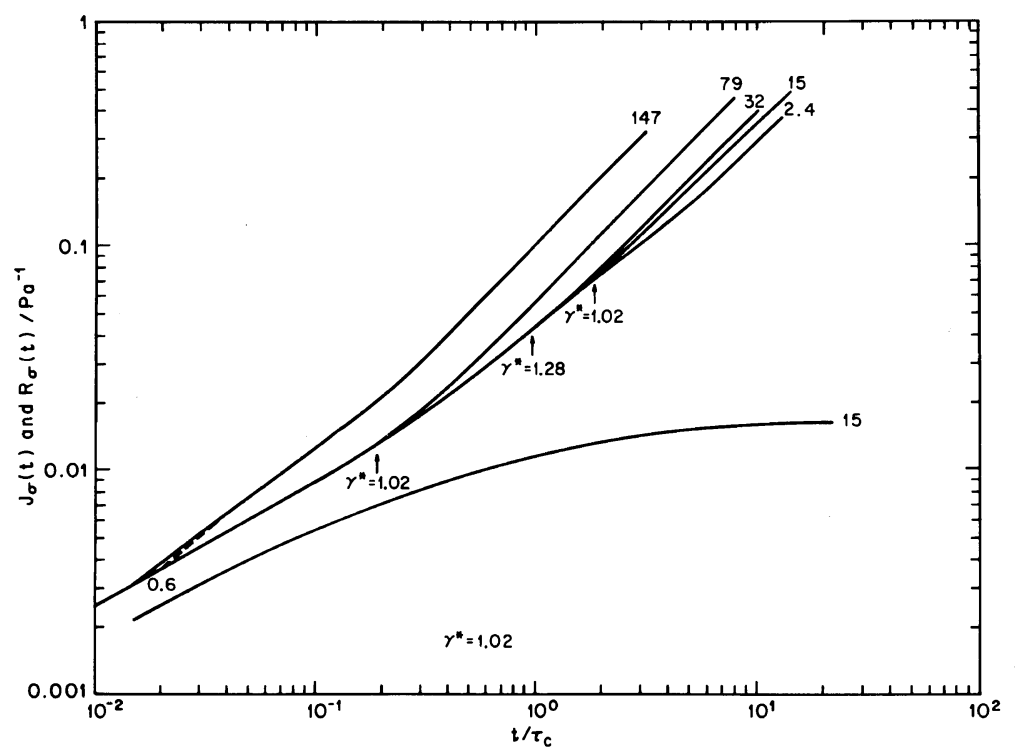

Figure 12. Creep $J_{\sigma}(t)$ and recoverable compliance $R_{\sigma}(t)$ versus $t / \tau_{\mathrm{c}}$ (with $\tau_{\mathrm{c}}=170 \mathrm{~s}$ ) for a nematic solution of PBT-53 $\left(w=32.3 \mathrm{~g} \mathrm{~kg}^{-1}, T=19.5^{\circ} \mathrm{C}\right)$. Values of shear stress $\sigma / \mathrm{Pa}$ used are given, along with the parameter $\gamma^{*}$.

behavior at small strain - i.e., no yield phenomenon is observed. For values of $\sigma$ such that $\partial \gamma(t) / \partial t$ at steady-state is in the range of $\kappa$ for which stress- growth experiments give $\eta_{\kappa} \approx \eta_{\mathrm{p}}$, the creep and stress-growth experiments give the same steadystate viscosity $\eta_{\mathrm{P}}$, as would normally be expected. 
Rheological Properties of Rodlike Polymers in Solution III.

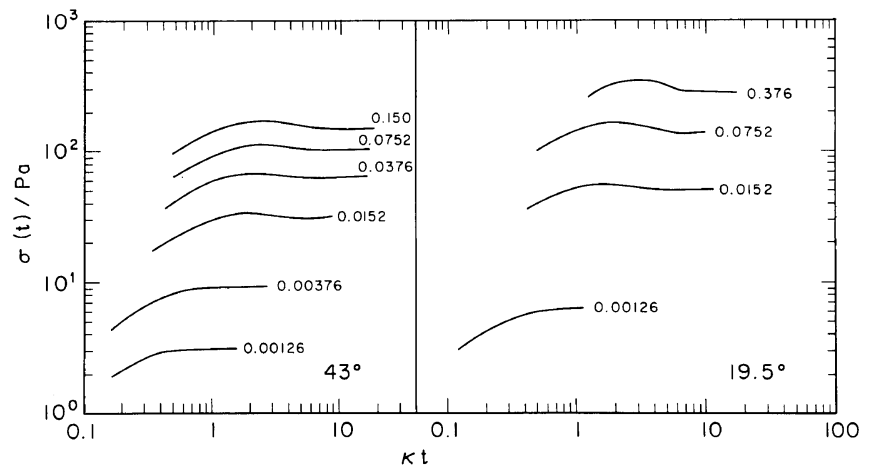

Figure 13. The stress $\sigma(t)$ in a stress-growth experiment versus the strain $\kappa t$ for a solution of PBT-53 $(w=$ $\left.32.3 \mathrm{~g} \mathrm{~kg}^{-1}\right)$ at temperatures for which the solution is isotropic $\left(43^{\circ} \mathrm{C}\right)$ and nematic $\left(19.5^{\circ} \mathrm{C}\right)$. Values of $\kappa /$ $\mathrm{s}^{-1}$ are indicated.

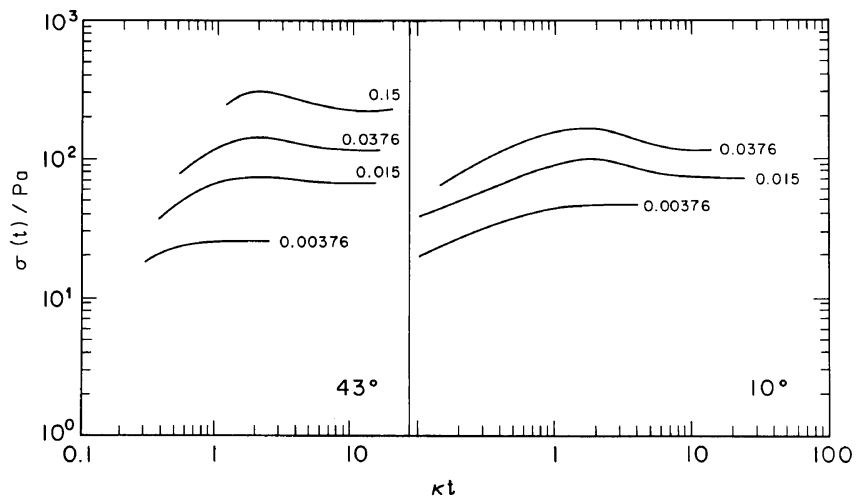

Figure 14. The stress $\sigma(t)$ in a stress-growth experiment versus the strain $\kappa t$ for a nematic solution of PBT-53 $\left(w=42.7 \mathrm{~g} \mathrm{~kg}^{-1}\right)$ at two temperatures.

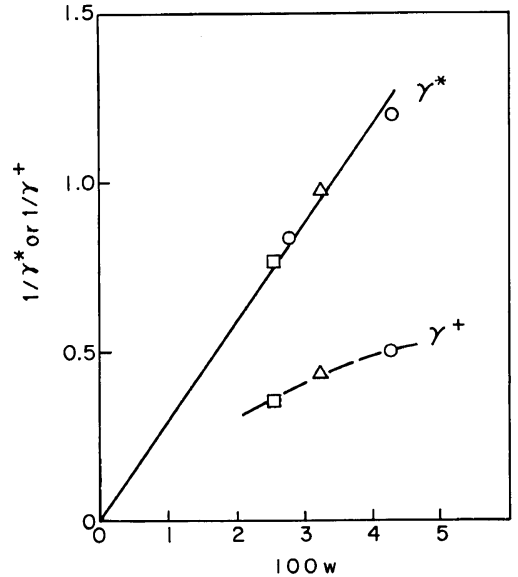

Figure 15. The critical strains $\gamma^{*}$ and $\gamma^{+}$plotted (as the inverse) versus the concentration $w$ for solutions of PBT53.
However, different behavior is obtained, for very small $\kappa$, for which stress-growth experiments give a larger steady-state viscosity, i.e., $\eta_{\kappa}>\eta_{\mathrm{P}}$. For example, with PBT-53 at $w=34.3 \mathrm{~g} \mathrm{~kg}^{-1}, \eta_{\kappa} \simeq 2.4 \eta_{\mathrm{P}}$ for $\kappa=3.8 \times 10^{-4} \mathrm{~s}^{-1}$ (i.e., $\eta_{\mathrm{P}} R_{0} \kappa=0.053$ ). For this sample, a creep experiment effected at $\sigma$ such that $\partial \gamma(t) / \partial t=3.8 \times 10^{-4} \mathrm{~s}^{-1}$ at steady-state resulted in a smaller steady-state viscosity, $\eta_{\kappa}=1.2 \eta_{\mathrm{P}}$. Values of $R_{0}$ determined after steady-state flow in these experiments were equivalent. In a creep experiment, $\partial \gamma(t) / \partial t$ is larger at small $t$ than at steady-state (i.e., large $t$ ). Thus, in the example cited the reduced $\eta_{\kappa}$ obtained in creep is in the range expected for a stress-growth experiment with $\kappa$ equal to $\partial \gamma(t) / \partial t$ obtained at small $t$ in the creep experiment. The quiescent, relaxed nematic fluids studied here are characterized by a texture such that locally, the axes of the rodlike chains are parallel, but with no 


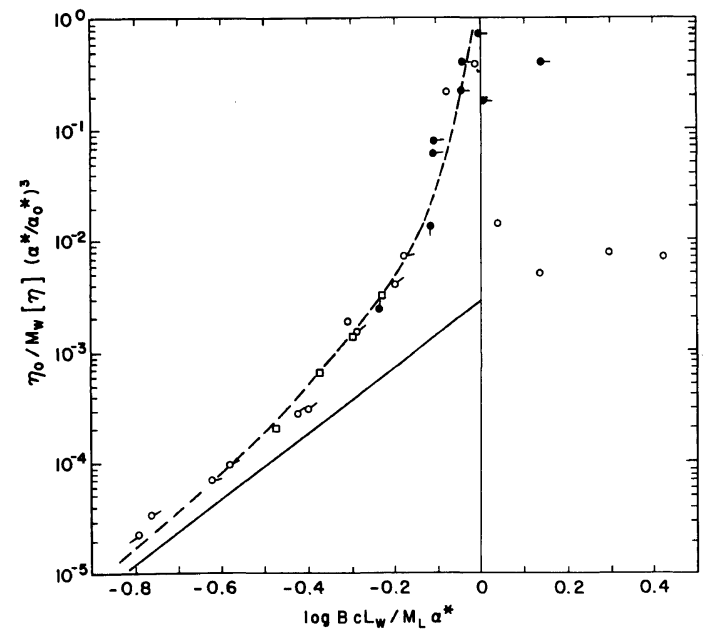

Figure 16. Left side: $\eta_{0} / M_{\mathrm{w}}[\eta]\left(a^{*} / a_{0}^{*}\right)^{3}$ versus $B c L_{\mathrm{w}} /$ $M_{\mathrm{L}} a^{*}$ for isotropic solutions of PBT-53, РBT-62 and other rodlike polymers (see Part II for identification of the symbols). Here $B / a^{*}$ is obtained by comparison of eq 8 with experiment (see Part II), and $B / a_{0}^{*}$ is an arbitrary constant. Right side: The same quantities for nematic solutions of PBT-62 (O) and PBT-53 (O-).

correlation among these axes over the entire sample. The behavior of $\eta_{x}$ at small $\kappa$ or $\sigma$ may be caused by effects on this texture. In particular, domains with the molecular axes of the rodlike chains oriented perpendicular to the flow plane may be depopulated with increasing $\kappa$ (or $\partial \gamma(t) / \partial t$ ), resulting in reduced $\eta_{\kappa}$. A similar suggestion has been made on the basis of flow birefringence data. ${ }^{11}$ If this view is correct, then comparison of $\eta_{\mathrm{P}}$, with $\eta_{\text {aniso }}$ may be inappropriate, since the latter is computed for a uniform fluid whereas $\eta_{\mathrm{P}}$ could be for fluid with the molecular axes oriented preferentially in the flow plane, so that one could have $\eta_{\mathbf{P}}<\eta_{\text {aniso, }}$, as the Miesowicz viscosities are unequal. ${ }^{12,13}$ The failure to observe an increase in $\eta_{\kappa}$ with decreasing $\kappa$ for some nematic fluids (e.g., PBT-53 with $w=42.7 \mathrm{~g}$ $\mathrm{kg}^{-1}$ ) may indicate suppressed domain structure (perhaps owing to incomplete relaxation of prior deformation) or insufficiently small $\kappa$ to observe the effect.

With increasing $\kappa$, the $\sigma(t)$ obtained in stressgrowth experiments display a maximum for strain $\kappa t$ about equal to a constant $\gamma^{+}$(independent of $\kappa$ ) for a given $c-\gamma^{+}$appears to decrease slowly with increasing $c$. This behavior is not unusual, and is observed with isotropic solutions of the polymers
Table III. Retardation and relaxation spectra

\begin{tabular}{|c|c|c|c|}
\hline$\lambda_{i} / \tau_{\mathrm{c}}$ & $R_{i} / R_{0}$ & $\tau_{i} / \tau_{\mathrm{c}}$ & $\eta_{i} / \eta_{0}$ \\
\hline \multicolumn{4}{|c|}{ PBT-53; $w=32.3 \mathrm{~g} \mathrm{~kg}^{-1}(\text { Nematic })^{\mathrm{a}}$} \\
\hline 8.30 & 0.262 & 8.59 & 0.037 \\
\hline 2.65 & 0.104 & 2.79 & 0.065 \\
\hline 1.04 & 0.312 & 1.40 & 0.266 \\
\hline \multirow[t]{2}{*}{0.193} & 0.170 & 0.353 & 0.323 \\
\hline & & 0.086 & 0.149 \\
\hline 0.042 & 0.111 & 0.0075 & 0.160 \\
\hline \multicolumn{4}{|c|}{ PBT-53; $w=29.4 \mathrm{~g} \mathrm{~kg}^{-1}$ (Isotropic) ${ }^{\mathrm{b}}$} \\
\hline 2.580 & 0.580 & 3.251 & 0.231 \\
\hline 0.210 & 0.280 & 0.494 & 0.480 \\
\hline \multirow[t]{2}{*}{0.0175} & 0.130 & 0.0615 & 0.092 \\
\hline & & 0.0010 & 0.092 \\
\hline \multicolumn{4}{|c|}{ PBT-53; $w=25.5 \mathrm{~g} \mathrm{~kg}^{-1}$ (Isotropic) } \\
\hline 5.248 & 0.430 & 5.732 & 0.094 \\
\hline 1.022 & 0.290 & 1.354 & 0.258 \\
\hline 0.240 & 0.174 & 0.381 & 0.253 \\
\hline \multirow[t]{2}{*}{$0.080_{5}$} & 0.035 & $0.098_{8}$ & 0.084 \\
\hline & & $0.025_{2}$ & 0.312 \\
\hline
\end{tabular}

${ }^{a} R_{0}, \eta_{0}$, and $\tau_{\mathrm{c}}$ replaced by values obtained from creep with shear stress $\sigma=14.6 \mathrm{~Pa}$, see the text; $\tau_{\mathrm{c}}=\eta_{0} R_{0}$.

b See Part II.

studied here, with $\gamma^{+}$being slightly larger for the latter. Similarly, with increasing $\sigma$, the $\gamma(t)$ obtained in creep experiments are given by $\sigma J_{0}(t)$ provided $\gamma(t)$ is smaller than a constant $\gamma^{*}$ (independent of $\sigma$ ) for a given $c$, with $\gamma(t)>\sigma J_{0}(t)$ for larger $\gamma(t)$. This is similar to behavior obtained with isotropic solutions of the rodlike chains, ${ }^{2}$ with $\gamma^{*}$ being somewhat smaller for the nematic solution than for the isotropic fluids. The combined behavior for stressgrowth and creep suggests that as with eq 3 , the rheological constitutive equation for nematic fluids may involve a critical strain criterion. The behavior at small $\kappa$ (or $\sigma$ ) suggests that eq 3 may not itself suffice.

Wtih the nematic fluids studied here, no proper linear viscoelastic behavior was observed, so that the $\tau_{\mathrm{i}}$ (or $\lambda_{\mathrm{i}}$ ) distribution could not be rigorously determined. Nevertheless, since for the nematic solutions, $\eta_{\kappa}$ and $R_{\kappa}$ are (nearly) independent of $\kappa$ over a reasonable range of $\kappa$ for $R_{0} \eta_{\mathrm{p}} \kappa$, in the range 0.1 to 1 , it is reasonable to compute (apparent) values of $R_{i}$ and $\lambda_{i}$ from data in $R_{\sigma}(t)$ for corresponding levels of $\sigma$. Values of $R_{i}$ and $\lambda_{i}$ determined for the data on $R_{\sigma}(t)$ in Figure 12 are given 


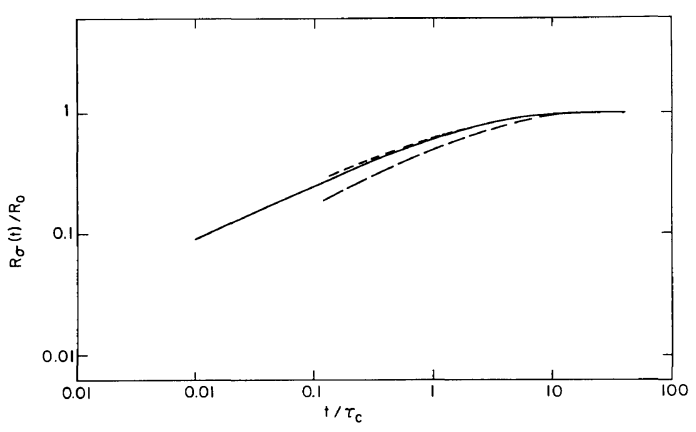

Figure 17. $R_{\sigma}(t) R_{0}$ versus $t / \tau_{\mathrm{c}}$ for two isotropic solutions of PBT-53 ( $w / \mathrm{g} \mathrm{kg}^{-1}$ equal to 25.5 and 29.4 , long and short dash, respectively) and a nematic solution $\left(w=32.3 \mathrm{~g} \mathrm{~kg}^{-1}, T=19.5^{\circ} \mathrm{C}\right.$, solid curve). For the latter, $\tau_{\mathrm{c}}$ is replaced by $\eta_{\kappa} R_{\kappa}$ and $R_{0}$ is replaced by $R_{\kappa}$. With the isotropic solutions, $R_{\sigma}(t)=R_{0}(t)$, and with nematic solution, $\sigma=14.6 \mathrm{~Pa}$.

in Table III - the data are normalized by the values of $R_{\kappa}$ and $\eta_{\kappa}$ obtained with the $\sigma$ used to determine $R_{\sigma}(t)$ (i.e., $\sigma=14.6 \mathrm{~Pa}$ ). Of course, the data in Table III reproduce $R_{\sigma}(t)$ in Figure 12. Their use to estimate $\eta_{\kappa}$ and $R_{\kappa}$ will be discussed below. The set of $\lambda_{i}$ for the nematic solution is similar in breadth to those obtained with an isotropic solution on PBT$53\left(w=29.4 \mathrm{~g} \mathrm{~kg}^{-1}\right)$, given in Table III for comparison, so that as shown in Figure 17, $R_{0}(t) / R_{0}$ for the latter is similar to $R_{\sigma}(t) / R_{\kappa}$ for the nematic solution. By comparison, $R_{0}(t) / R_{0}$ and the $\lambda_{\mathrm{i}}$ set for a solution of PBT-53 $\left(w=25.5 \mathrm{~g} \mathrm{~kg}^{-1}\right)$ are noticeably different

As shown in Part II, for isotropic solutions, $\eta_{\kappa} / \eta_{0}$ and $R_{\kappa} / R_{0}$ may be represented in terms of eq 5 and 6 given the parameters $\eta_{i}$ and $\tau_{\mathrm{i}}$ (or $R_{i}$ and $\lambda_{i}$ ). With these fluids (as with flexible chain polymers ${ }^{4}$ ) over a wide range $\tau_{\mathrm{c}} \kappa$ (with $\tau_{\mathrm{c}}=\eta_{0} R_{0}$ ), the approximations

$$
\begin{aligned}
& \eta_{\kappa} \simeq\left[\eta_{0}(t)\right]_{\kappa t=1} \\
& R_{\kappa} \simeq\left[R_{0}(t)\right]_{\kappa t=1}
\end{aligned}
$$

provide direct correspondence between linear and nonlinear properties. In particular, with a narrow distribution of $\tau_{i}, R_{\kappa}$ is essentially independent of $\tau_{\mathrm{c}} \kappa$ over a wide range of $\tau_{\mathrm{c}} \kappa$. With the isotropic solutions studied here, the distribution of $\tau_{i}$ is reasonably broad, so that $R_{\kappa}$ decreases markedly with increasing $\tau_{\mathrm{c}} \kappa$, as discussed in Part II, in good agreement with eq 13. Similar behavior is shown in Figure 7 for a nematic solution, where $R_{\sigma}(t)$ for a low $\sigma$ corresponding to the range for which $\eta_{\kappa} \approx \eta_{\mathrm{P}}$ and $R_{\kappa}=R_{0}$. As mentioned above, for the isotropic solutions the functions $\eta_{\kappa} / \eta_{0}$ and $R_{\kappa} / R_{0}$ versus $\tau_{\mathrm{c}} \kappa$ are independent of $T$ for a given material. In the interests of completeness, reduced curves for isotropic solutions of PBT-53 and 62 given in Parts I and II are included in Figures 5 and 6. For PBT-53, $\eta_{\kappa} / \eta_{0}$ and $R_{\kappa} / R_{0}$ are fitted well by eq 5 and 6 , respectively, using $\eta_{i}$ and $\tau_{i}$ determined by linear viscoelastic studies. As may be seen in Figure 6, the decrease of $R_{\kappa} / R_{0}$ with increasing $\tau_{\mathrm{c}} \kappa$ is more pronounced with the isotropic solutions of PBT-62 than those of PBT-53, indicating a broader $\tau_{\mathrm{i}}$ (or $\lambda_{i}$ ) distribution with the PBT-62 solutions.

The set of $\tau_{i}$ and $\eta_{i}$ given in Table III for the nematic solution with $w=32.3 \mathrm{~g} \mathrm{~kg}^{-1}$ were computed from the $\lambda_{i}$ and $R_{i}$ given in Table III. The $\tau_{i}$ and $\eta_{i}$ were calculated using the expressions for linear viscoelasticity, despite the nonlinearity discussed in the preceding. The functions $\eta_{\kappa} / \eta_{\mathrm{P}}$ and $R_{\kappa} /$ $R_{0}$ versus $R_{0} \eta_{\mathrm{p}} \kappa$ computed using these $\tau_{i}$ and $\eta_{i}$ in eq 5 and 6 are shown in Figure 7-these curves were computed with $\gamma^{\prime}=1.0$ and $\gamma^{\prime \prime}=2.4$, in accord with the experimental data. The computed $R_{\kappa} / R_{0}$ are in good accord with the experimental $\eta_{k} / \eta_{\mathrm{P}}$; the computed $R_{\kappa} / R_{0}$ tend to lie $20-40 \%$ above the measured values for large $R_{0} \eta_{\mathrm{P}} \kappa$. Apparently, the quasilinear treatment of the data used to estimate $\tau_{i}$ and $\eta_{i}$, etc., is useful in the rage studied here, provided $R_{0} \eta_{\mathrm{p}} \kappa$ is at least as large as the value needed to obtain the "plateau" in $\eta_{\kappa}$ (e.g., $R_{0} \eta_{\mathrm{p}} \kappa>0.1$ for PBT-53 with $w=32.3 \mathrm{~g} \mathrm{~kg}^{-1}$ ).

It may be noted that the treatment given above would not lead to negative first normal stress $v_{\kappa^{\prime}}^{(1)}$ as has been reported for an ordered solution of $\operatorname{poly}(\gamma-$ benzyl glutamate), (PBG). Thus, with the singleintegral equation used to obtain eq 5 and 6 , the reduced parameter $N_{\kappa}^{(1)}=v_{\kappa}^{(1)} / 2\left(\kappa \eta_{\kappa}\right)^{2}$ is given by

$$
\eta_{\kappa}^{2} N_{\kappa}^{(1)}=\sum \eta_{i} \tau_{i}\left(1-q_{\kappa, i} P_{\kappa i}\right)
$$

where $P_{k, i}$ is a function of $g_{i}$ and $\alpha$ (see Part II). With eq $14, N_{\kappa}^{(1)}$ is always positive. Data were obtained in our laboratory on a solution of PBLG in cresol for which negative values of $N_{\kappa}^{(1)}$ were, obtained $^{14}$ - the solution was provided by R. S. Porter. The results for $\eta_{\kappa}$, given in Figure 18 were in good agreement with data reported by Porter and Kiss over the range of $\kappa$ for which the data overlapped. Data obtained here at lower $\kappa$ did not become independent of $\kappa$. Values of $R_{\kappa}$ were found to decrease smoothly with increasing $\kappa$, through the 


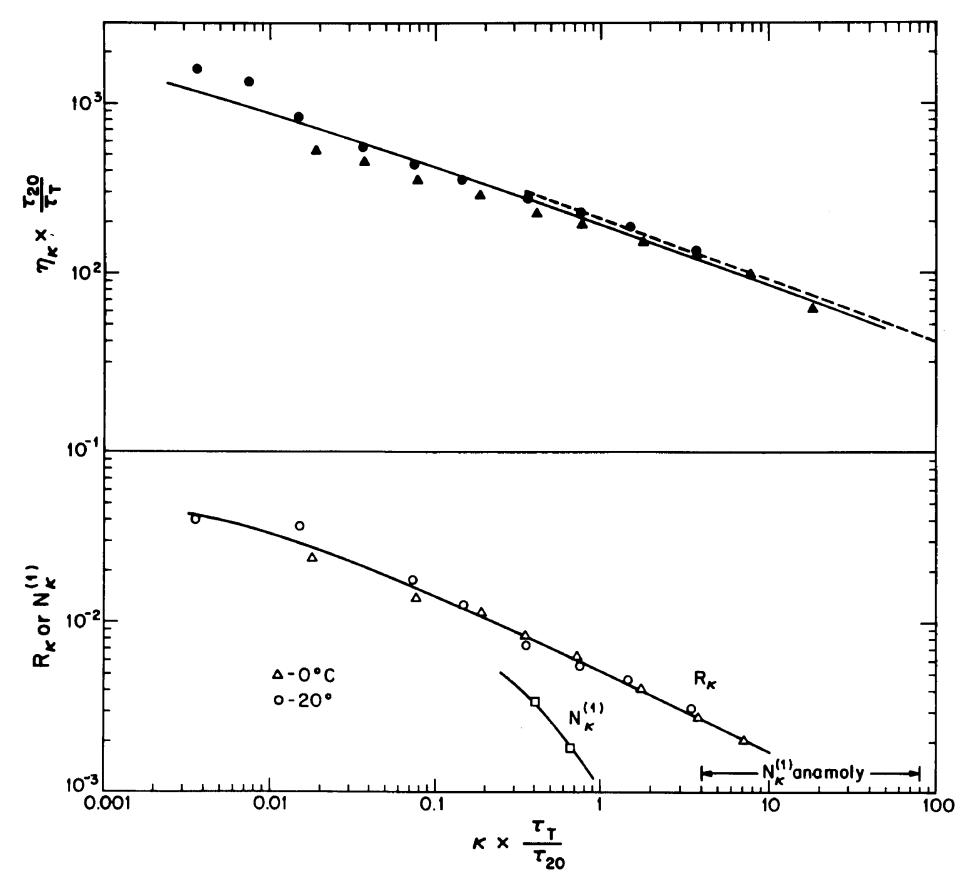

Figure 18. $\eta_{\kappa}\left(\tau_{20} / \tau_{\mathrm{T}}\right), R_{\kappa}$ and $N_{\kappa}^{(1)} \operatorname{versus}\left(\tau_{\mathrm{T}} / \tau_{20}\right) \kappa$ for an anisotropic solution of poly $(\gamma$-benzyl glutamate $)$ in cresol for $T / \mathrm{K}$ equal to $273(\triangle, \mathbf{\Delta})$ and $293(\bigcirc, \bullet)$. The dashed line and the data on $N_{\kappa}{ }^{(1)}$ are from reference 12. The factor $\tau_{\mathrm{T}} / \tau_{20}$ is unity for $T=293 \mathrm{~K}$ and chosen to superpose data on $R_{\kappa}$ versus $\left(\tau_{\mathrm{T}} / \tau_{20}\right) \kappa$ for $T=273 \mathrm{~K}$. The range denoted " $N_{\kappa}^{(1)}$ anomaly" designates the range for negative $N_{\kappa}^{(1)}$ according to ref 14.

range of $\kappa$ for which $N_{\kappa}^{(1)}$ reported to be negative. On the basis of the behavior observed for $\eta_{\kappa}$ and $R_{\kappa}$, one can speculate that the $\tau_{i}$ (or $\lambda_{i}$ ) distribution would be very broad for the PBG solution. With eq 5 and $14, N_{\kappa}^{(1)}$ may become very small with $\eta_{\kappa} / \eta_{0}$ not too much less than unity with some $\tau_{i}$-distribution, but $N_{\kappa}^{(1)}$ will always be positive.

With a PBT-62 solution studied for which $c$ was slightly less than $c_{\mathrm{c}}$ so that the quiescent fluids were (macroscopically) isotropic, the data on $\eta_{\kappa}$ versus $\kappa$ did not display any range of $\kappa$ for which $\eta_{\kappa}$ was independent of $\kappa$. Rather, as shown in Figures 4 and $10, \eta_{\kappa}$ decreased slowly with increasing $\kappa$ at small $\kappa$. For these solutions, the decrease in $R_{\kappa}$ with increasing $\kappa$ was much smaller than observed with isotropic solutions (see Figure 10). This behavior, which is found only over a narrow range of conditions with $c$ close to, but less than $c_{\mathfrak{c}}$, may be caused by a tendency for the isotropic solutions to develop orientational order characteristic of the nematic state under the influence of a shear stress, so that $\eta_{\kappa}$ decreases slowly with increasing $\kappa$.
As shown in Figure 11, the data on $\eta_{\kappa} / \eta_{0}$ versus $\tau_{\mathrm{c}} \kappa$ for solution with $c$ near $c_{\mathrm{c}}$ for the temperature of the measurement do not superpose with each other and differ from data on the isotropic solutions at lower $c$. For example, with PBT-62, the data on $\eta_{\kappa} /$ $\eta_{0}$ versus $\tau_{\mathrm{c}} \kappa$ for the solution with $w / \mathrm{g} \mathrm{kg}^{-1}$ equal to 30.0 and 34.3 do not superpose over the temperature range studied. In terms of eq 5 or 6 , it appears that the distribution of $\tau_{i}$ sharpens with decreasing $T$ for this solution. The behavior of $R_{\kappa} / R_{0}$, which becomes nearly independent of $\tau_{0} \kappa$ at low $T$, is also consistent with an effectively narrowed distribution of $\tau_{i}$.

Acknowledgement. It is a pleasure to acknowledge partial support for this study from the Polymers Program, Division of Materials Research, National Science Founation, and the Materials Laboratory, Wright-Patterson Air Force Base. 
Rheological Properties of Rodlike Polymers in Solution III.

\section{REFERENCES}

1. S.-G. Chu, S. Venkatraman, G. C. Berry, and Y. Einaga, Macromolecules, 14, 939 (1981).

2. S. Venkatraman, G. C. Berry, and Y. Einaga, J. Polym. Sci., Polym. Phys. Ed., submitted.

3. J. D. Ferry, "Viscoelastic Properties of Polymers," 3rd ed, Wiley, New York, 1980.

4. K. Nakamura, C.-P. Wong, and G. C. Berry, J. Polym. Sci., Polym. Phys. Ed., 22, 1119 (1984)

5. M. Doi, J. Phys. (Paris), 36, 607 (1975).

6. M. Doi and S. F. Edwards, J. Chem. Soc. Faraday Trans. 2, 74, 560 (1978).

7. J. A. Odell, E. D. T. Atkins, and A. Keller, J. Polym.
Sci., Polym. Lett. Ed., 21, 289 (1983).

8. K. F. Wissbrun, J. Rheol., 25, 619 (1981).

9. M. Doi, J. Polym. Sci., Polym. Phys. Ed., 19, 229 (1981).

10. G. C. Berry, P. Metzger Cotts, and S.-G. Chu, Br. Polym. J., 13, 47 (1981).

11. T. Asada, H. Muramatsu, R. Watanabe, and S. Onogi, Macromolecules, 13, 867 (1980).

12. G. Marrucci, Mol. Cryst. Liq. Cryst. (Lett.), 72, 153 (1982).

13. N. Kuzuu and M. Doi, J. Phys. Soc. Jpn., 52, 3486 (1983); ibid., 53, 1031 (1984).

14. G. Kiss and R. S. Porter, J. Polym. Sci., Polym. Symp., 65, 193 (1978). 